\title{
SOLUTION OF MECHANICAL SYSTEMS WITH UNCERTAINTY PARAMETERS USING IFEA
}

The paper presents a non-traditional computational approach for structural analysis with uncertainties in material, geometry and load parameters. Uncertainties are introduced as bounded possible values - intervals. The main goal is to propose algorithms for interval computations on FEM models suggested by authors. An application of the chosen approaches is presented - the first one, a simple combination of only inf-values or only sup-values; the second one presents full combination of all inf-sup values; the third one uses the optimisation process as a tool for finding out a inf-sup solution and last one is Monte Carlo technique as a comparison tool.

Keywords: uncertain structural parameters, MATLAB, Monte Carlo, interval arithmetic

\section{Introduction}

In the last years there has been an increased interest in the modeling and analysis of engineering systems under uncertainties. To obtain reliable results for the solution of engineering problems, exact values for the parameters of the model equations should be available. Really, however, those values can often not be provided, and the models usually exhibit a rather high degree of uncertainty. Computational mechanics, for example, entails uncertainties in geometry, material and load parameters as well as in the model itself and in the analysis procedure too. For that reason, the responses, such as displacements, stresses, resonant frequency, or other dynamic characteristics, will usually exhibit any degree of uncertainty. It means that the obtained result using one specific value as a most significant value for uncertain parameter cannot be considered to be representative for the whole spectrum of possible results.

It is generally known that probabilistic modeling and statistical analysis is well-established for modeling of mechanical systems with uncertainties. In addition, a number of non-probabilistic computational techniques have been proposed, e.g. fuzzy set theory [ 1 , $2,10]$, interval approach $[2,6]$, imprecise probabilities [9], etc. The growing interest in these approaches originated from criticism of the credibility of probabilistic approach when input dates are insufficient [10]. It is argued that the new non-probabilistic treatments could be more appropriate in modeling of the vagueness.

The uncertainty is assumed to be unknown but bounded with lower and upper bounds. The uncertainties in the model parameters, model inputs etc. can then be taken into account by interval numbers derived from experimental data or expert knowledge. By this technique, the complete information about the uncertainties in the model may be included and one can demonstrate how these uncertainties are processed through the calculation procedure in MATLAB.

\section{Fundamental Principles of Interval Arithmetic}

Interval arithmetic was developed by R. E. Moore [5] while studying the propagation and control of truncation and round off error using floating point arithmetic on a digital computer. Moore was able to generalize this work into the arithmetic independent of machine considerations [5, 7].

In this approach, an uncertain number is represented by an interval of real numbers. An interval number $[3,5,7]$ is a closed set $\mathbf{R}$ that includes the possible range of an unknown real number where $\mathbf{R}$ denotes the set of real numbers. Therefore, a real interval is a set of the form

$$
\mathbf{x}=[\underline{x}, \bar{x}]=\{x \in \mathbf{R}: \underline{x} \leq x \leq \bar{x}\},
$$

where $\underline{x}$ and $\bar{x}$ are the lower (infimum) and upper (supremum) bounds of the interval number $\mathbf{x}$ respectively, and the bounds are elements of $\mathbf{R}$ with $\underline{x} \leq \bar{x}$. Definition of real intervals and operations with intervals could be found in a number of references [2, $3,6,7,10]$. Let's define basic properties of interval number that have been inbuilt in INTLAB [8]:

- the midpoint of $\mathbf{x}: \quad \operatorname{mid}(x)=\breve{x}=\frac{1}{2}(\underline{x}+\bar{x})$,

- the radius of $\mathbf{x}: \quad \operatorname{rad}(\mathbf{x})=\frac{1}{2}(\bar{x}-\underline{x})$,

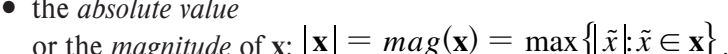

\footnotetext{
* Milan Vasko, Milan Saga

Department of Applied Mechanics, Faculty of Mechanical Engineering, University of Zilina, E-mail: milan.vasko@fstroj.uniza.sk
} 
- the magnitude of $\mathbf{x}: \quad \operatorname{mig}(\mathbf{x})=\min \{|\tilde{x}|: \tilde{x} \in \mathbf{x}\}$.

Given $\mathbf{x}[\underline{x}, \bar{x}]$ and $\mathbf{y}=[\underline{y}, \bar{y}]$, the four elementary operations are defined by

$$
\begin{aligned}
& \mathbf{x}+\mathbf{y}=[\underline{x}+\underline{y}, \bar{x}+\bar{y}], \\
& \mathbf{x}-\mathbf{y}=[\underline{x}-\bar{y}, \bar{x}-\underline{y}], \\
& x \times y=[\min \{\underline{x y}, \underline{x} \bar{y}, \bar{x} \underline{y}, \overline{x y}\}, \max \{\underline{x y}, \underline{x} \bar{y}, \bar{x} \underline{y}, \overline{x y}\}], \\
& \mathbf{x} \div \mathbf{y}=\mathbf{x} \times 1 / \mathbf{y}, \text { where } 1 / \mathbf{x}=[1 / \bar{x}, 1 / \underline{x}] \text { if } \underline{x}>0, \bar{x}<0 .
\end{aligned}
$$

For the elementary interval operations, division by an interval containing zero is not defined. It is often useful to remove this restriction to give what is called extended interval arithmetic [3] Extended interval arithmetic leads to the following rules. If $\mathbf{x}[\underline{x}, \bar{x}]$ and $\mathbf{y}=[\underline{y}, \bar{y}]$ with $\underline{y} \leq 0 \leq \bar{y}$ and $\underline{y}<\bar{y}$, then the rules for division are as follows

$$
\mathbf{x} / \mathbf{y}=\left\{\begin{array}{ll}
{[\bar{x} / y, \infty]} & \text { if } \bar{x} \leq 0 \text { and } \bar{y}=0 \\
{[-\infty, \bar{x} / \bar{y}] \cup[\bar{x} / \underline{y}, \infty]} & \text { if } \bar{x} \leq 0 \text { and } \underline{y}<0<\bar{y} \\
{[-\infty, \bar{x} / \bar{y}]} & \text { if } \bar{x} \leq 0 \text { and } \underline{y}=0 \\
{[-\infty, \infty]} & \text { if } \underline{x}<0<\bar{x} \\
{[-\infty, \underline{x} / \underline{y}]} & \text { if } \underline{x} \geq 0 \text { and } \bar{y}=0 \\
{[-\infty, \bar{x} / \underline{y}] \cup[\underline{x} / \bar{y}, \infty]} & \text { if } \underline{x} \geq 0 \text { and } \underline{y}<0<\bar{y} \\
{[\underline{x} / \bar{y}, \infty]} & \text { if } \underline{x} \geq 0 \text { and } \underline{y}=0
\end{array}\right\} .
$$

For further rules for extended interval arithmetic see $[2,3,7$, $10]$.

\section{Computational Approaches in Interval Matrix Analysis}

Considering uncertain parameters in interval form, we'll realize comparison study of the few basic matrix operations:

- solution of the linear equations system (INTLAB function - verifylnss),

- solution of the eigenvalue problem (INTLAB function - verifyeig).

The alternative way of the interval arithmetic [8] can be the following numerical approaches:

- so called Monte Carlo computational technique,

- Inf-Sup simple analysis (COM1),

- Inf-Sup full analysis (COM2),

- Inf-Sup optimising analysis (OPT).

The comparison was realised through the use of midpoin residual vector $\mathbf{r}_{\text {Midpoint }}$ and radius residual vector $\mathbf{r}_{\text {Residual }}$ expressed in $\%$, e.g.

$$
\begin{aligned}
& \mathbf{r}_{\text {Midpoint }}=\left|\frac{\operatorname{mid}\left(\mathbf{y}_{\text {Intlab }}\right)-\operatorname{mid}\left(\mathbf{y}_{M C}\right)}{\operatorname{mid}\left(\mathbf{y}_{\text {Intlab }}\right)}\right| \cdot 100 \% \\
& \mathbf{r}_{\text {Radius }}=\left|\frac{\operatorname{rad}\left(\mathbf{y}_{\text {Intlab }}\right)-\operatorname{rad}\left(\mathbf{y}_{M C}\right)}{\operatorname{rad}\left(\mathbf{y}_{\text {Intlab }}\right)}\right| \cdot 100 \%
\end{aligned}
$$

Monte Carlo method (MC) is a time consuming but reliable solution. Various combinations of the uncertain parameter deterministic values are generated and after the subsequent solution in the deterministic sense we obtain a complete set of results processed in an appropriate manner. Infimum and supremum calculation is following

$\inf (F)=$ minof all results $F\left(p_{i}\right)$, where $i=1, \ldots, m$ and $m \approx 5000 \div 100000$,

$\operatorname{sub}(F)=$ minof all results $F\left(p_{i}\right)$, where $i=1, \ldots, m$ and

$m \approx 5000 \div 100000$.

The second method (COM1), i.e. the solution evaluation in marginal values of interval parameters has its physical meaning for many engineering problems. We consider an approach where the extreme output values are obtained by the application of the extreme parameter values on input. That means that the infimum/ supremum is obtained using the deterministic analysis for infimum or supremum of input uncertain parameters. Inf-Sup calculation is

$$
\inf (F)=\min \text { of }[F(p), F(\bar{p})] \text { and }
$$$$
\sup (F)=\max \text { of }[F(p), F(\bar{p})] \text {. }
$$

The third approach (COM2), which is also based on the set of deterministic analyses appears as the more suitable one. The marginal interval parameter values are considered again but the infimums and supremums are also combined. The method provides satisfying results and can be marked as reliable, even if there is still a doubt about the existence of the extreme solution for the uncertain parameter inner values. A solution for two interval numbers $p_{1}=\left\langle a_{1} b_{1}\right\rangle$ and $p_{2}=\left\langle a_{2} b_{2}\right\rangle$ may be found in the following computational way

and

$\inf (F)=\min$ of $\left[F\left(a_{1} a_{2}\right), F\left(a_{1} b_{2}\right), F\left(b_{1} a_{2}\right), F\left(b_{1} b_{2}\right)\right]$

$\sup (F)=\max$ of $\left[F\left(a_{1} a_{2}\right), F\left(a_{1} b_{2}\right), F\left(b_{1} a_{2}\right), F\left(b_{1} b_{2}\right)\right]$

The last numerical method of the infimum and supremum solution searching using the optimization techniques (OPT) is proposed by the authors as an alternative to the first and to the third method. It should eliminate a big amount of analyses in the first method and also eliminates the problem with the possibility of the infimum and supremum existence inside the interval parameters for the deterministic values. Computational process for the two interval numbers $p_{1}=\left\langle a_{1} b_{1}\right\rangle$ and $p_{2}=\left\langle a_{2} b_{2}\right\rangle$ may be found as follows

and

$\inf (F)=F\left(\mathbf{p}_{O P T}\right)$ i.e. find $\mathbf{p}_{O P T}$ so that $F\left(\mathbf{p}_{O P T}\right) \rightarrow \min$.

$\sup (F)=F\left(\mathbf{p}_{O P T}\right)$ i.e. find $\mathbf{p}_{O P T}$ so that $F\left(\mathbf{p}_{O P T}\right) \rightarrow \max$ 
The authors used also the interval arithmetic principles implemented in INTLAB [8] as another computing tool. However, the overestimate effect mentioned above for the significant uncertainties causes considerable problems and the possibilities of INTLAB using are therefore very restricted. INTLAB using makes sense particularly for simple problems because of the results obtaining speed.

\section{Testing example 1}

Let's applied the presented methods into the solution of the following system

$$
\left[\begin{array}{cc}
\langle 0.1\rangle & 1 \\
-2 & \langle-1.2\rangle
\end{array}\right] \cdot\left[\begin{array}{l}
y_{1} \\
y_{2}
\end{array}\right]=\left[\begin{array}{c}
\langle 0.2\rangle \\
0
\end{array}\right]
$$

The solution is shown in Fig. 1 and used confrontation methods are summarized in Tab. 1.

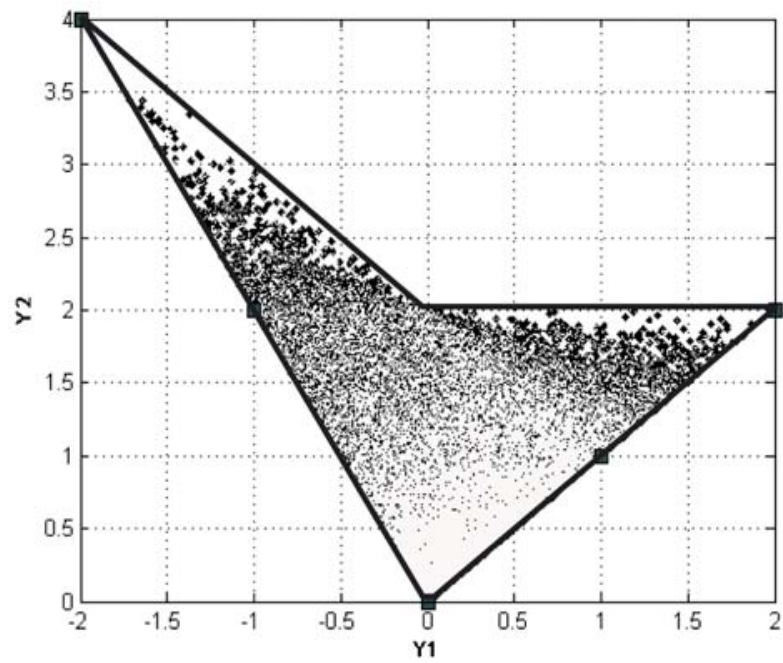

Fig. 1 Results map of interval linear equations system

Results obtained by proposed numerical methods

Tab. 1

\begin{tabular}{|c|c|c|c|c|c|c|}
\hline & $\begin{array}{c}\text { Exact } \\
\text { solution }\end{array}$ & $\begin{array}{c}1^{\text {st }} \\
\text { method } \\
\text { MC }\end{array}$ & $\begin{array}{c}2^{\text {nd }} \\
\text { method } \\
\text { COM1 }\end{array}$ & $\begin{array}{c}3^{\text {rd }} \\
\text { method } \\
\text { COM2 }\end{array}$ & $\begin{array}{c}4^{\text {th }} \\
\text { method } \\
\text { OPT }\end{array}$ & $\begin{array}{c}5^{\text {th }} \\
\text { method } \\
\text { INT }\end{array}$ \\
\hline Y1 & $\langle-22\rangle$ & $\langle-1.951 .94\rangle$ & $\langle-11\rangle$ & $\langle-22\rangle$ & $\langle-20\rangle$ & $\langle-4.784 .78>$ \\
\hline Y2 & $\langle 04\rangle$ & $\langle 03.91\rangle$ & $\langle 02\rangle$ & $\langle 04\rangle$ & $\langle 04\rangle$ & $\langle-5.1$ 5.1> \\
\hline
\end{tabular}

In general, the interval system of equations solving may be a complicated problem (mainly for large dimension, [7]). Good information about the solution set is obtained using the Monte Carlo method. The other methods determine only the marginal values with bigger or smaller inaccuracies. The $1^{\text {st }}$ and the $3^{\text {rd }}$ used methods are particularly suitable for the equations systems solving according to the experiences of the authors. In case of the fourth method application (the optimization method) there is a problem with formulation of the appropriate test function which would properly describe searching of multiple inf or sup solutions. INTLAB usage appears to be unsuitable because the parameter uncertainty is rather strong.

\section{Testing example 2}

Let's compare proposal interval computational methods to solve the following eigenvalues problem

a) with a small signification of the parameters uncertainty, e.g.

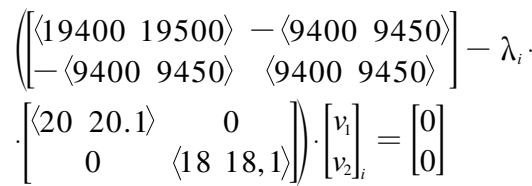

b) with a larger signification of the parameters uncertainty, e.g.

$$
\begin{aligned}
& \left(\left[\begin{array}{cccc}
\langle 19400 & 21400\rangle & -\langle 9400 & 10400\rangle \\
-\langle 9400 & 10400\rangle & \langle 9400 & 10400\rangle
\end{array}\right]-\lambda_{i} .\right. \\
& \left.\cdot\left[\begin{array}{ccc}
\langle 2025\rangle & 0 \\
0 & \langle 18 & 22\rangle
\end{array}\right]\right) \cdot\left[\begin{array}{l}
v_{1} \\
v_{2}
\end{array}\right]_{i}=\left[\begin{array}{l}
0 \\
0
\end{array}\right]
\end{aligned}
$$

Inf-sup results were compiled into tab. 2. Graphic presentation of the results is shown in Fig. 2.

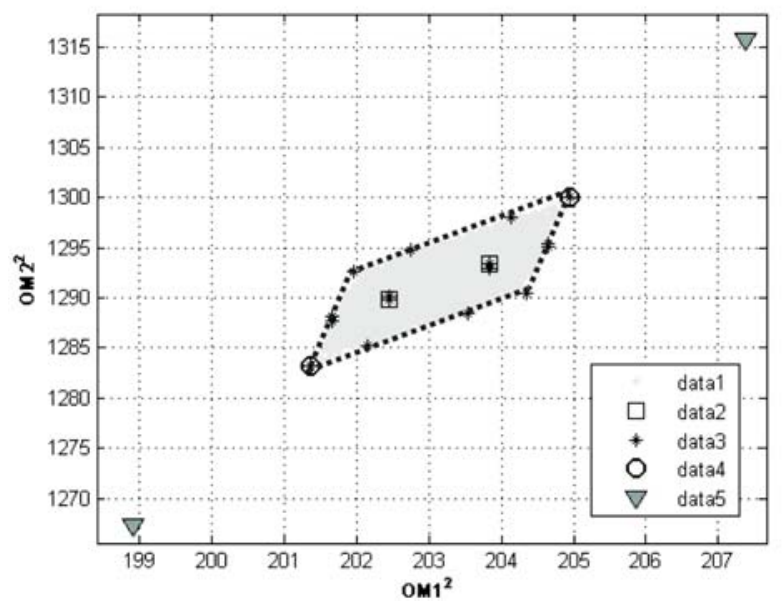

Fig. 2 Solution of the set eigenvalues with small uncertainties

data 1 - Monte Carlo analysis

data2 - COM1, evaluation in marginal values (only inf or only sup) data3 - COM2, evaluation for all marginal values-all combinations data4 - OPT, inf and sup searching using optimizing technique application

data5 - direct application of the interval arithmetic using INTLAB ---- - boundary of the all possible solutions set

The solution of an eigenvalues problem as a frequent task of solid mechanics has demonstrated facilities of particular approaches. The Monte Carlo method usually gives good information about the solution set. Other methods determine only marginal values. For the eigenvalues analysis of the systems with interval parame- 
ters the authors recommend according to their experiences to use particularly the $1^{\text {st }}, 3^{\text {rd }}$ and $4^{\text {th }}$ method. All of them give satisfying results but especially the $3^{\text {rd }}$ and $4^{\text {th }}$ ones appear to be the most suitable. From the effectiveness perspective the third method can be considered as the best even if there is a risk of losing the inner interval number solutions. The usage of the second and the fifth method (INTLAB) is determined by the uncertainty importance (the interval size). As it is presented, all the methods are acceptable in case of a minor uncertainty (solution A).

\section{Interval finite elements analysis (IFEA)}

It is common knowledge that the finite element method (FEM) $[4,6,10]$ is a very popular tool for complicated structural analysis. The ability to predict the behavior of a structure under static or dynamic loads is not only of great scientific value, it is also very useful from an economical point of view. A reliable FE analysis could reduce the need for prototype production and therefore significantly reduce the associated design validation cost.

It is sometimes very difficult to define a reliable FE model for realistic mechanical structures when a number of its physical properties are uncertain. Particularly, in the case of FE analysis, the mechanical properties of the used materials are very hard to predict, and therefore an important source of uncertainty. Reliable validation can only be based on an analysis which takes into account all uncertainties that could cause this variability. It is the aim of this part to incorporate most important uncertainties in FE analysis.

According to character of the uncertainty we can define a structural uncertainty (geometrical and material parameters) and uncertainty in load (external forces, etc.). The structural uncertainty parameters are usually written into vector $[\mathbf{x}]=[\mathbf{x}, \overline{\mathbf{x}}]$ and the interval static (time independent) FE analysis may be formulated as follows

$$
\mathbf{K}([\mathbf{x}]) \cdot \mathbf{u}=\mathbf{f}([\mathbf{x}]) \text { or }[\underline{\mathbf{K}}, \overline{\mathbf{K}}] \cdot[\underline{\mathbf{u}}, \overline{\mathbf{u}}]=[\underline{\mathbf{f}}, \overline{\mathbf{f}}],
$$

where $\mathbf{K}, \overline{\mathbf{K}}$ are the infimum and supremum matrices of the stiffness matrix $\mathbf{K}, \underline{\mathbf{u}}, \overline{\mathbf{u}}$ are the infimum and supremum vectors of the displacements vector $[\mathbf{u}], \mathbf{f}, \overline{\mathbf{f}}$ are the infimum and supremum vectors of the loading vector $\mathbf{f}$. Considering a dynamic conservative system, the interval modal and spectral matrices can be obtained using the solution of

$$
\begin{aligned}
& \left\lfloor\mathbf{K}(\mathbf{x})-\lambda_{j} \cdot \mathbf{M}(\mathbf{x})\right\rfloor \cdot \mathbf{v}_{j}=0 \text { or } \\
& \left.\left(\lfloor\underline{\mathbf{K}}, \overline{\mathbf{K}}\rfloor-\left\lfloor\underline{\lambda_{j}}, \overline{\lambda_{j}}\right] \cdot[\underline{\mathbf{M}}, \overline{\mathbf{M}}]\right) \cdot \mid \underline{v_{j}}, \overline{v_{j}}\right\rfloor=0,
\end{aligned}
$$

where $\underline{\lambda_{j}}, \overline{\lambda_{j}}$ and $\underline{v_{j}}, \overline{v_{j}}$ are the $j$-th eigenvalue with a corresponding eigenvector $\mathbf{K}, \overline{\mathbf{K}}, \mathbf{M}, \overline{\mathbf{M}}$ are naturally the infimum and supremum of the mass and stiffness matrices. The application of classical interval arithmetic for FE analysis is very limited. Its "overestimating" grows with a problem size (dimension of system matrices) and really has no physical foundation. Therefore, it is effective to apply the previous numerical methods.

The application of the Monte Carlo method in IFEA may be realized as follows

\section{Static analysis}

1. step: generation of the random matrix (uniform distribution)

$$
\mathbf{X}_{M C}=\left[x_{1}, \ldots, x_{m}\right], \quad(m \approx 5000 \div 100000)
$$

2. step: solution of

$$
\mathbf{U}_{M C}=\left[\mathbf{K}\left(x_{1}\right)^{-1} \cdot \mathbf{f}\left(x_{1}\right), \ldots, \mathbf{K}\left(x_{m}\right)^{-1} \cdot \mathbf{f}\left(x_{m}\right)\right]
$$

3. step:

$$
\begin{array}{ll}
\text { - infimum calculation } & \underline{u}_{i}=\inf \left(i^{\text {th }} \text { row of } \mathbf{U}_{M C}\right) \rightarrow \underline{\mathbf{u}}, \\
\text { - supremum calculation } & \bar{u}_{i}=\sup \left(i^{\text {th }} \text { row of } \mathbf{U}_{M C}\right) \rightarrow \overline{\mathbf{u}} .
\end{array}
$$

\section{Eigenvalues analysis}

1. step: generation of the random matrix (uniform distribution)

$$
\mathbf{X}_{M C}=\left[x_{1}, \ldots, x_{m}\right], \quad(m \approx 5000 \div 100000)
$$

2. step: solution of

$$
\lambda_{j \_M C} \rightarrow\left[\mathbf{K}\left(x_{j}\right)-\lambda_{j \_M C} \cdot \mathbf{M}\left(x_{j}\right)\right] \cdot \mathbf{v}_{j}=0 \text { for } j=1, \ldots, m
$$

3. step:

- infimum calculation of the i-th eigenvalue

$\underline{\lambda_{i}}=\inf \left(i^{\text {th }}\right.$ row of $\left.\lambda_{M C}\right)$,

- supremum calculation of the i-th eigenvalue

\begin{tabular}{|c|c|c|c|c|c|c|}
\hline & & $\begin{array}{c}1^{\mathrm{st}} \\
\text { method }\end{array}$ & $\begin{array}{c}2^{\text {nd }} \\
\text { method }\end{array}$ & $\begin{array}{c}3^{\text {rd }} \\
\text { method }\end{array}$ & $\begin{array}{l}4^{\text {th }} \\
\text { method }\end{array}$ & $\begin{array}{c}5^{\text {th }} \\
\text { method }\end{array}$ \\
\hline \multirow{2}{*}{ A } & $\lambda_{1}$ & $<201203\rangle$ & $\langle 202.3202 .5\rangle$ & $<201203>$ & $<201203>$ & $<199206>$ \\
\hline & $\lambda_{2}$ & $\langle 1284$ 1296> & $\langle 1289$ 1290> & $\langle 1283$ 1296> & $\langle 1283$ 1296> & $\langle 1268$ 1312> \\
\hline \multirow{2}{*}{ B } & $\lambda_{1}$ & $\langle 167220\rangle$ & $\langle 181$ 202〉 & $\langle 164$ 223> & $\langle 164223\rangle$ & $\langle 95$ 287〉 \\
\hline & $\lambda_{2}$ & $\langle 1059$ 1415> & $\langle 1147$ 1290> & $\langle 10391425\rangle$ & $\langle 1038$ 1425> & $\langle 6001827\rangle$ \\
\hline
\end{tabular}

$\bar{\lambda}_{i}=\sup \left(i^{\text {th }}\right.$ row of $\left.\lambda_{M C}\right)$.

In the case of implementation of COM1 numerical approach to IFEA is as follows

Static analysis

- infimum calculation $\quad \underline{\mathbf{u}}=\mathbf{K}(\underline{\mathbf{x}})^{-1} \cdot \mathbf{f}(\underline{\mathbf{x}})$

- supremum calculation $\quad \overline{\mathbf{u}}=\mathbf{K}(\overline{\mathbf{x}})^{-1} \cdot \mathbf{f}(\overline{\mathbf{x}})$.

Results obtained by proposed numerical methods 
Eigenvalues analysis

- infimum calculation

$$
\underline{\boldsymbol{\lambda}} \rightarrow[\mathbf{K}(\underline{\mathbf{x}})-\underline{\boldsymbol{\lambda}} \cdot \mathbf{M}(\underline{\mathbf{x}})] \cdot \underline{\mathbf{v}}=0
$$

- supremum calculation

$\overline{\boldsymbol{\lambda}} \rightarrow[\mathbf{K}(\overline{\mathbf{x}})-\overline{\boldsymbol{\lambda}} \cdot \mathbf{M}(\overline{\mathbf{x}})] \cdot \overline{\mathbf{v}}=0$

COM1 doesn't give correct results every time. Using COM2 we can obtain more correct results. Its computational process for IFEA is

\section{Static analysis}

1. step: calculation of realizations matrix $X_{2}$, i.e. $2^{\text {n }}$ inf-sup combinations, ( $\mathrm{n}$ - number of uncertain system parameters),

$\mathbf{X}_{\text {Сом } 2}=\left[\mathbf{x}_{1}, \ldots, \mathbf{x}_{m}\right],\left(m=2^{n}\right)$

2. step: solution of

$$
\mathbf{U}_{\text {сом } 2}=\left[\mathbf{K}\left(\mathbf{x}_{1}\right)^{-1} \cdot \mathbf{f}\left(\mathbf{x}_{1}\right), \ldots, \mathbf{K}\left(\mathbf{x}_{m}\right) \cdot \mathbf{f}\left(\mathbf{x}_{m}\right)\right]
$$

3. step:

- infimum calculation $\quad \underline{\mathbf{u}_{i}}=\inf \left(i^{\text {th }}\right.$ row of $\left.\mathbf{U}_{M C}\right) \rightarrow \underline{\mathbf{u}}$,

- supremum calculation $\quad \overline{\mathbf{u}}_{i}=\sup \left(i^{\text {th }}\right.$ row of $\left.\mathbf{U}_{M C}\right) \rightarrow \overline{\mathbf{u}}$.

Eigenvalues analysis

1. step: calculation of realizations matrix $X_{2}$, i.e. $2^{n}$ inf-sup combinations, ( $\mathrm{n}$ - number of uncertain system parameters),

$\mathbf{X}_{\text {сом } 2}=\left[\mathbf{x}_{1}, \ldots, \mathbf{x}_{m}\right],\left(m=2^{n}\right)$

2. step: solution of

$\boldsymbol{\lambda}_{j_{-} \text {Сом } 2} \rightarrow\left[\mathbf{K}\left(\mathbf{x}_{j}\right)-\boldsymbol{\lambda}_{j_{-} \text {Сом2 }} \cdot \mathbf{M}\left(\mathbf{x}_{j}\right)\right] \cdot \mathbf{v}_{j}=0$ for $j=1, \ldots, m$

3. step:

- infimum calculation of the i-th eigenvalue

$\underline{\lambda_{i}}=\inf \left(i^{\text {th }}\right.$ row of $\left.\boldsymbol{\lambda}_{\text {сом } 2}\right)$,

- supremum calculation of the i-th eigenvalue

$\bar{\lambda}_{i}=\sup \left(i^{\text {th }}\right.$ row of $\left.\lambda_{\text {Сом2 }}\right)$.

Generally, the infimum or supremum are not found in only bound points (COM1, COM2) but also in inner domain of the solution set (OPT). To find inf-sup solution using the OPT approach means to solve the optimizing problem described as follows

\section{Static analysis}

- infimum calculation $u_{i}\left(\mathbf{x}_{\text {OPT }}\right) \rightarrow$ minimize value $i^{\text {th }}$ member of $\left[\mathbf{K}(\mathbf{x})^{-1} \cdot \mathbf{f}(\mathbf{x})\right]$

- supremum calculation $\bar{u}_{i}\left(\mathbf{x}_{\text {OPT }}\right) \rightarrow$ maximize value $i^{\text {th }}$ member of $\left[\mathbf{K}(\mathbf{x})^{-1} \cdot \mathbf{f}(\mathbf{x})\right]$.

\section{Eigenvalues analysis}

- infimum calculation of the $i$-th eigenvalue $\lambda_{i}\left(\mathbf{x}_{O P T}\right) \rightarrow$ minimize value of $\lambda_{i}$ for eq. $\left[\mathbf{K}(\mathbf{x})^{-1}-\lambda_{i} \cdot \mathbf{M}(\mathbf{x})\right] \cdot \mathbf{v}_{i}=0$,

- supremum calculation of the $i$-th eigenvalue $\bar{\lambda}_{i}\left(\mathbf{x}_{O P T}\right) \rightarrow$ minimize value of $\lambda_{i}$ for eq. $\left[\mathbf{K}(\mathbf{x})^{-1}-\lambda_{i} \cdot \mathbf{M}(\mathbf{x})\right] \cdot \mathbf{v}_{i}=0$,

It should be noted that the searching process can be realized by a comparison of an optimizing method (e.g. Nelder-Mead simplex algorithm) or by using a genetic algorithm as a robust tool of global optimization.

Testing example 3 - Stress interval analysis of the truss structure For the following research purpose on the interval finite element model computing, the truss structure shown in Fig. 3 was analyzed, where the geometry of the structure is presented. The truss structure was loaded by uncertain forces F1 and F2. The certain model parameters are defined as follows

- element mass density $\rho=2700 \mathrm{~kg} \cdot \mathrm{m}^{-3}$,

- nodal concentrated mass (nodes $33,34,35,36) \mathrm{m}=50 \mathrm{~kg}$.

\section{Stress interval analysis of the truss structure}

Let's assume the following uncertain model parameters:

- Young's modulus $\quad E=\langle 0.95 \quad 1.05\rangle \cdot 2 \cdot 10^{11} \mathrm{~Pa}$,

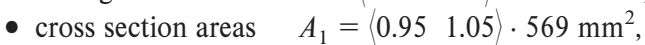

$A_{2}=\langle 0.951 .05\rangle \cdot 691 \mathrm{~mm}^{2}$,

$A_{3}=\langle 0.951 .05\rangle \cdot 1550 \mathrm{~mm}^{2}$,

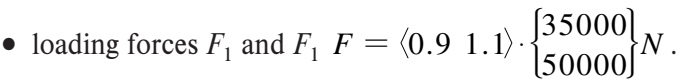

The input uncertain parameters in the vector form will be defined for the further analyses as follows

$$
\mathbf{x}=\left[E, A_{1}, A_{2}, A_{3}, F_{1}, F_{2}\right]
$$

The purpose of this study will be to compare the efficiency and exactness of the proposed methods MC, COM1, COM2 and OPT. The results of the MC analysis will be considered as reference values and will be used for the construction of the solution map. In the case of MC method, 5000 random inputs were generated; they were evaluated and properly processed to inf-sup solutions.

The maximal stress values calculated by the particular methods for the truss loaded by maximal stress (truss no. 15) achieved the following values:

$$
\begin{aligned}
& \langle-343-255\rangle \quad(\mathrm{MC}) \\
& \left\langle\sigma_{15}\right\rangle=\begin{array}{ll}
\langle-343-255\rangle & (\mathrm{COM} 1) \\
\langle-343-255\rangle \quad(\mathrm{OPT})
\end{array} \\
& \langle-343-255\rangle \text { (COM2) }
\end{aligned}
$$

The results are obtained from the final arrangement of the solution set applying the searching algorithm for the infimum and supremum as follows:

$$
\begin{aligned}
& \inf =\min (\max |\sigma(\mathbf{x})|), \\
& \sup =\max (\max |\sigma(\mathbf{x})|) .
\end{aligned}
$$

If the COM1, COM2 and OPT methods are compared with the MC method, it can be observed that:

- the COM1 method is less appropriate and is not recommended for this kind of analyses,

- the OPT method provides comparable, in some cases even better results than the MC method and what is very important, it does not need so many analyses as the MC method, 
- the disadvantage of MC and OPT methods is a problem with finding a solution in the solution map corners,

- the COM2 method does not necessarily have to give exact results, but from the perspective of the number of performed analyses, it is more effective than the MC or OPT methods and it can "find" the solutions in the solution map corners,

- the previous considerations lead to the recommendation to combine COM2 and MC or OPT methods.

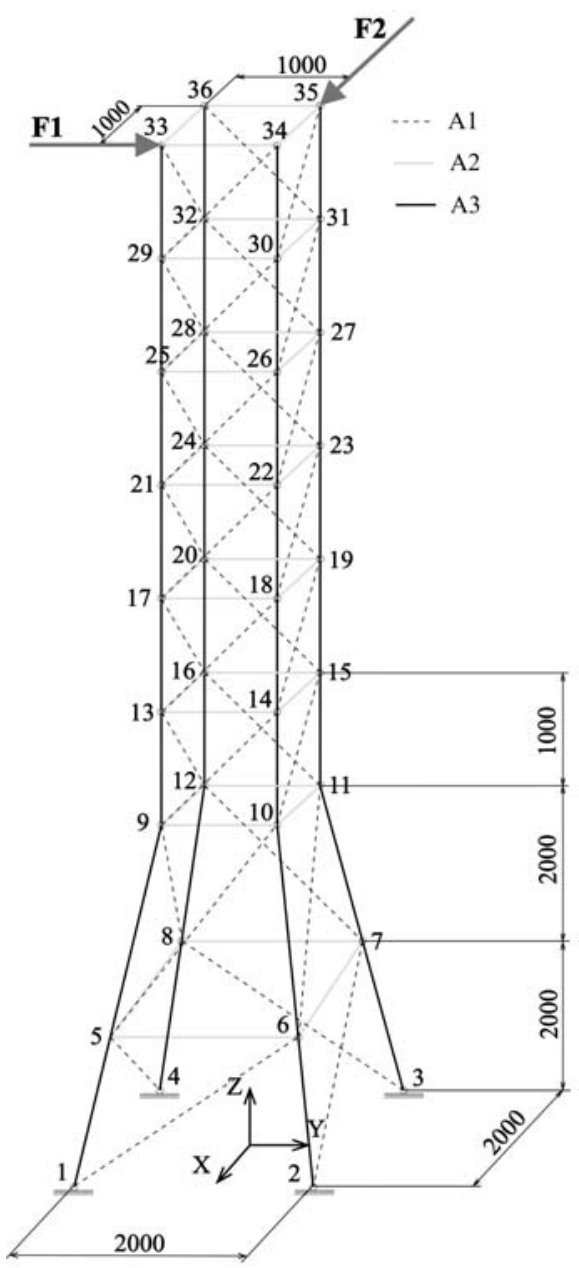

Fig. 3 Analyzed truss structure

Errors in midpoints of the stress

Tab. 3 in critical element No. 15

\begin{tabular}{|c|c|c|c|c|c|c|c|}
\hline \multirow{4}{*}{ Stress } & MC & \multicolumn{2}{|c|}{ COM1 } & \multicolumn{2}{c|}{ OPT } & \multicolumn{2}{c|}{ COM2 } \\
\cline { 2 - 7 } & $\begin{array}{c}\text { Refe- } \\
\text { rence } \\
\text { mid- } \\
\text { point }\end{array}$ & $\begin{array}{c}\text { Mid- } \\
\text { point }\end{array}$ & $\begin{array}{c}\text { Error } \\
{[\%]}\end{array}$ & $\begin{array}{c}\text { Mid- } \\
\text { point }\end{array}$ & $\begin{array}{c}\text { Error } \\
{[\%]}\end{array}$ & $\begin{array}{c}\text { Mid- } \\
\text { point }\end{array}$ & $\begin{array}{c}\text { Error } \\
{[\%]}\end{array}$ \\
\hline $\begin{array}{c}\sigma_{15} \\
{[\mathrm{MPa}]}\end{array}$ & -299 & -299.5 & 0.17 & -299.5 & 0.17 & -298.5 & 0.17 \\
\hline
\end{tabular}

Errors in radiuses of the stress

Tab. 4 in critical element No. 15

\begin{tabular}{|c|c|c|c|c|c|c|c|}
\hline \multirow{2}{*}{ Stress } & MC & \multicolumn{2}{|c|}{ COM1 } & \multicolumn{2}{c|}{ OPT } & \multicolumn{2}{c|}{ COM2 } \\
\cline { 2 - 7 } & $\begin{array}{c}\text { Refe- } \\
\text { rence } \\
\text { mid- } \\
\text { point }\end{array}$ & Radius & $\begin{array}{c}\text { Error } \\
{[\%]}\end{array}$ & Radius & $\begin{array}{c}\text { Error } \\
{[\%]}\end{array}$ & Radius & $\begin{array}{c}\text { Error } \\
{[\%]}\end{array}$ \\
\hline $\begin{array}{c}\sigma_{15} \\
{[\mathrm{MPa}]}\end{array}$ & 44 & 14.5 & 67 & 34.5 & 21.6 & 44.5 & 1.1 \\
\hline $\begin{array}{c}\text { Appli- } \\
\text { cation } \\
\text { possibi- } \\
\text { lity }\end{array}$ & good & \multicolumn{2}{|c|}{ bad } & limited & \multicolumn{2}{|c|}{ good } \\
\hline
\end{tabular}

Testing example 4 - Interval eigenvalue solving of fourdrinier structures

For the following research purposes on the interval finite element model computing, the frame structure shown on the Fig. 4 was analyzed. The certain material parameters are defined as follows element mass density $\rho=2700 \mathrm{~kg} \cdot \mathrm{m}^{-3}$ and Young's modulus $E=2 \cdot 10^{11} \mathrm{~Pa}$.

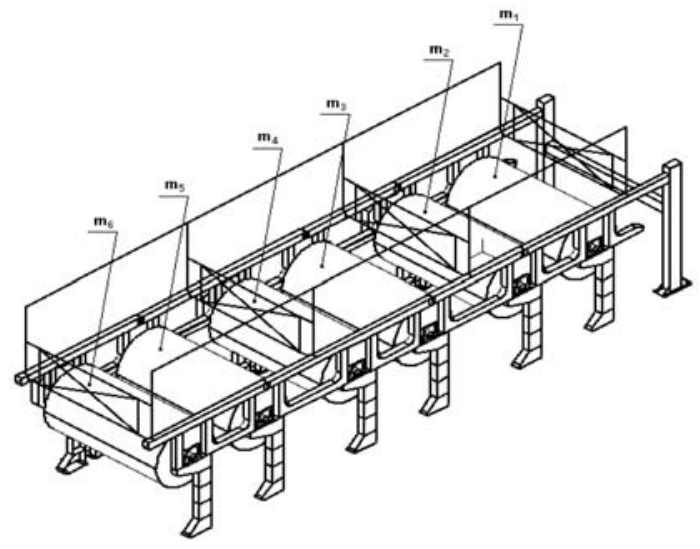

Fig. 4 Analyzed fourdrinier structure

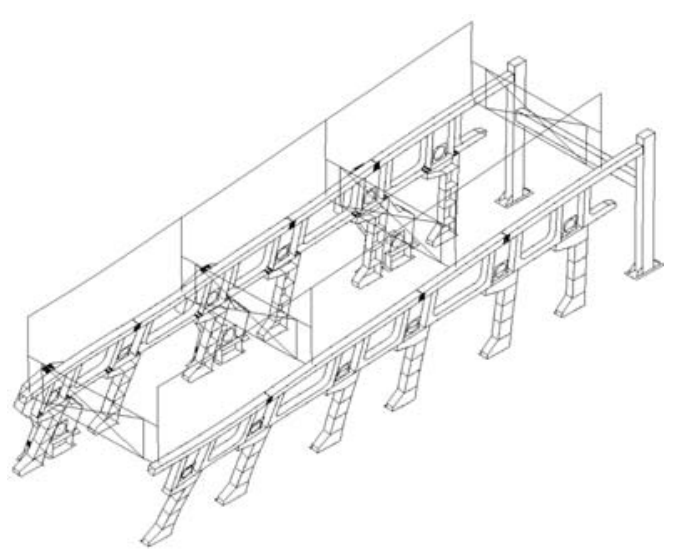

Fig. 5 Modal shape for natural freq. $f_{I}=3.69 \mathrm{~Hz}$ 


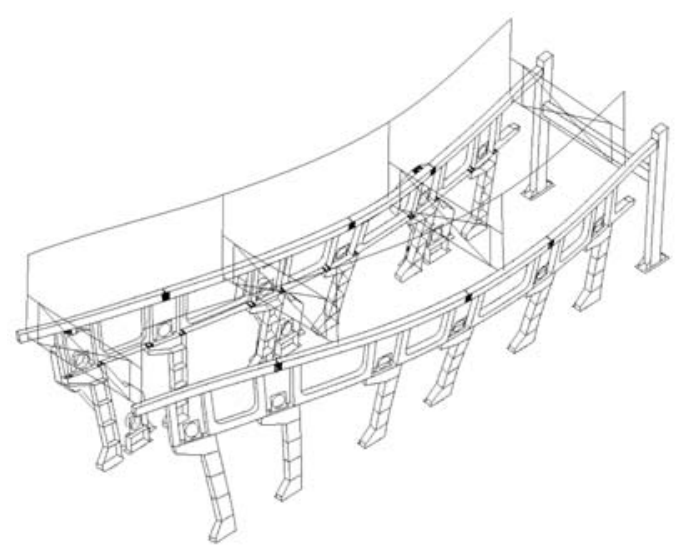

Fig. 6 Modal shape for natural freq. $f_{2}=4.17 \mathrm{~Hz}$

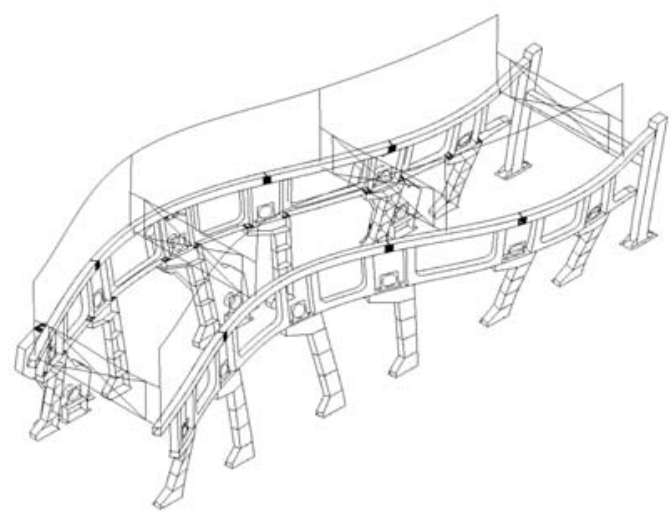

Fig. 7 Modal shape for natural freq. $f_{3}=5.56 \mathrm{~Hz}$

The purpose of this study is to compare the efficiency and exactness of the proposed methods MC, COM1, COM2 and OPT. The results of the MC analysis are considered as the reference values and are used for the construction of the solution map. The interval modal and spectral analysis of the identical structure was realized assuming the following uncertain model parameters (mass of cylinders, Fig. 4):

$$
\mathbf{x}=\left[m_{1}, m_{2}, m_{3}, m_{4}, m_{5}, m_{6}\right], \text { where } m_{1 \div 6} \in\langle 0,2200\rangle[\mathrm{Pa}] .
$$

The solution will now consider only the analyses of the first three interval natural frequencies $f_{1}, f_{2}$ and $f_{3}$ (See Figs. 5, 6, 7).
In the case of MC method, 2000 random inputs were generated, evaluated and properly processed to inf-sup solutions

$$
\inf =\min \left(f_{1}(\mathbf{x})\right), \sup =\min \left(f_{2}(\mathbf{x})\right) .
$$

The interval solution results are summarized in Tabs. 5, 6, and 7.

Errors in midpoints of natural frequencies

Tab. 6

\begin{tabular}{|c|c|c|c|c|c|c|c|}
\hline \multirow{2}{*}{$\begin{array}{c}\text { Freq. } \\
\text { no. }\end{array}$} & \begin{tabular}{c} 
MC \\
\cline { 2 - 8 } \\
$\begin{array}{c}\text { Refe- } \\
\text { rence } \\
\text { point }\end{array}$
\end{tabular} & \multicolumn{2}{|c|}{ COM1 } & \multicolumn{2}{c|}{ OPT } & \multicolumn{2}{c|}{ COM2 } \\
point & $\begin{array}{c}\text { Error } \\
{[\%]}\end{array}$ & $\begin{array}{c}\text { Mid- } \\
\text { point }\end{array}$ & $\begin{array}{c}\text { Error } \\
{[\%]}\end{array}$ & $\begin{array}{c}\text { Mid- } \\
\text { point }\end{array}$ & $\begin{array}{c}\text { Error } \\
{[\%]}\end{array}$ \\
\hline $\mathrm{f}_{1}[\mathrm{~Hz}]$ & 3.41045 & 3.4250 & 0.427 & 3.42185 & 0.334 & 3.4249 & 0.424 \\
\hline $\mathrm{f}_{2}[\mathrm{~Hz}]$ & 3.8521 & 3.8835 & 0.815 & 3.8395 & 0.327 & 3.88355 & 0.816 \\
\hline $\mathrm{f}_{3}[\mathrm{~Hz}]$ & 5.1571 & 5.165 & 0.153 & 5.0065 & 2.92 & 5.165 & 0.153 \\
\hline
\end{tabular}

Errors in radiuses of natural frequencies

Tab. 7

\begin{tabular}{|c|c|c|c|c|c|c|c|}
\hline \multirow{2}{*}{$\begin{array}{c}\text { Freq. } \\
\text { no. }\end{array}$} & \begin{tabular}{c} 
MC \\
\cline { 2 - 8 }
\end{tabular} $\begin{array}{c}\text { Refe- } \\
\text { rence } \\
\text { mid- } \\
\text { point }\end{array}$ & Radius & $\begin{array}{c}\text { Error } \\
{[\%]}\end{array}$ & Radius & $\begin{array}{c}\text { Error } \\
{[\%]}\end{array}$ & Radius & $\begin{array}{c}\text { Error } \\
{[\%]}\end{array}$ \\
\hline $\mathrm{f}_{1}[\mathrm{~Hz}]$ & 0.21575 & 0.253 & 17.265 & 0.25015 & 15.944 & 0.2532 & 17.358 \\
\hline $\mathrm{f}_{2}[\mathrm{~Hz}]$ & 0.2295 & 0.2895 & 26.144 & 0.2459 & 7.146 & 0.28995 & 26.340 \\
\hline $\mathrm{f}_{3}[\mathrm{~Hz}]$ & 0.3405 & 0.396 & 16.300 & 0.238 & 30.103 & 0.3965 & 16.446 \\
\hline
\end{tabular}

The graphical representation for the all computational approaches is shown in Fig. 8. This figure presents a map of the input data set for each of the methods. The "iterative" map with the infimum and supremum of the first two natural frequencies obtained using the suggested methods is shown in Fig. 9. Due to the effectiveness of the COM2 and OPT methods a short comparison of their iteration history was done. Figs. 10 and 11 present iteration history of the cylinder masses and Figs. 12 and 13 map the iteration process of the natural frequencies $f_{1}, f_{2}$.

\begin{tabular}{|c|c|c|c|c|}
\hline Freq. no. & MC & COM1 & OPT & COM2 \\
\hline $\mathrm{f}_{1}[\mathrm{~Hz}]$ & $\left\langle\begin{array}{lll}3.1947 & 3.6262\end{array}\right.$ & $\left\langle\begin{array}{lll}3.1720 & 3.6780\end{array}\right.$ & $\left\langle\begin{array}{lll}3.1717 & 3.6720\end{array}\right.$ & $\left\langle\begin{array}{lll}3.1717 & 3.6781\end{array}\right\rangle$ \\
\hline $\mathrm{f}_{2}[\mathrm{~Hz}]$ & $\langle 3.6226 \quad 4.0816\rangle$ & $\langle 3.5940 \quad 4.1730\rangle$ & $\left\langle\begin{array}{lll}3.5936 & 4.0854\end{array}\right.$ & $\left\langle\begin{array}{lll}3.5936 & 4.1735\end{array}\right.$ \\
\hline $\mathrm{f}_{3}[\mathrm{~Hz}]$ & $\langle 4.8166 \quad 5.4976\rangle$ & $\langle 4.7690 \quad 5.5610\rangle$ & $\langle 4.7685 \quad 5.2445\rangle$ & $\left\langle\begin{array}{lll}4.7685 & 5.5615\end{array}\right.$ \\
\hline
\end{tabular}




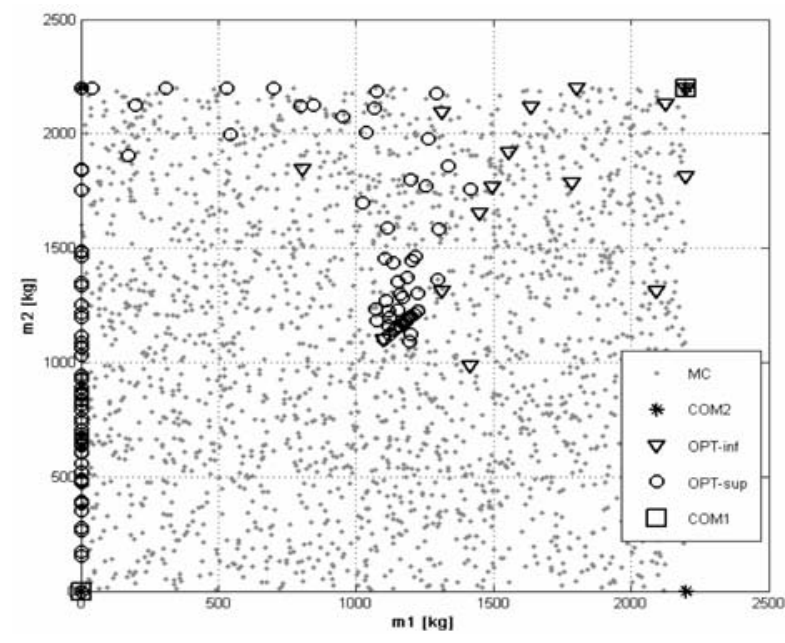

Fig. 8 Mapping of the generated input data for applied methods

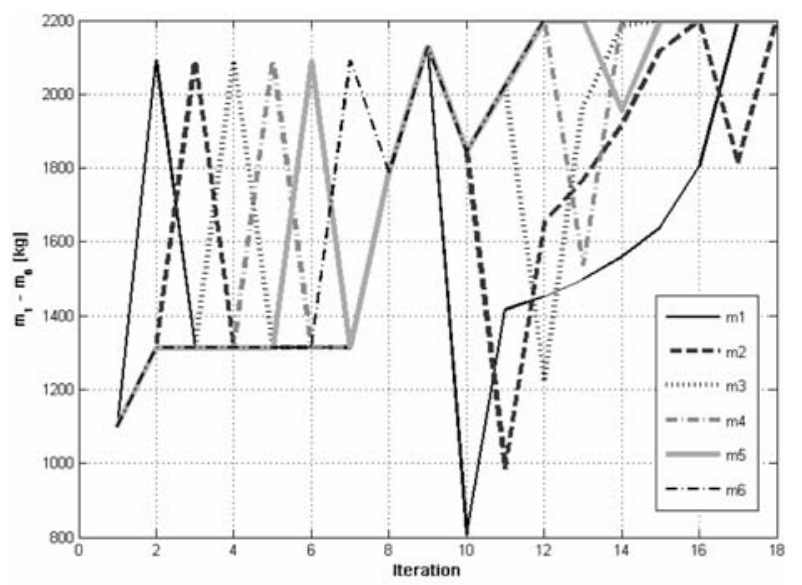

Fig. 10 History of the iteration process for cylinder masses - COM2

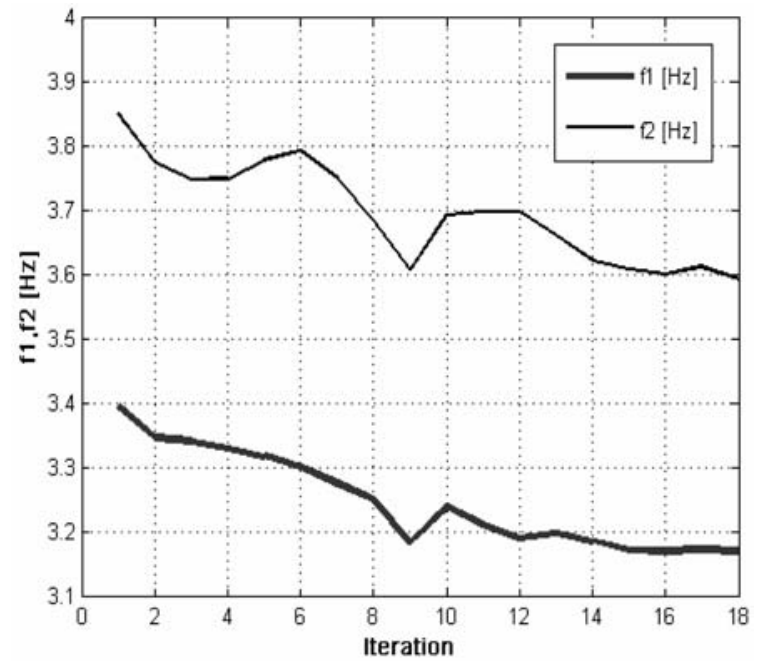

Fig. 12 History of the iteration process for natural frequences $f_{1}, f_{2}-$ COM2

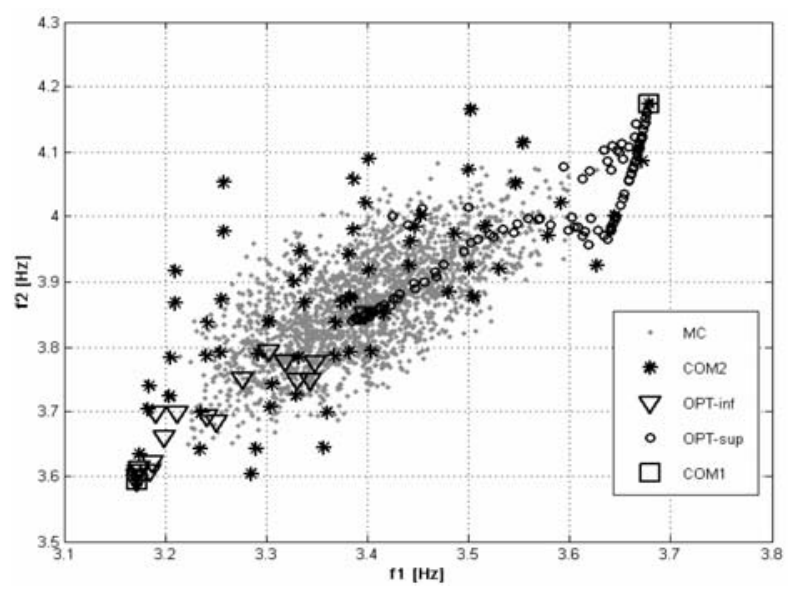

Fig. 9 Mapping of the solution history for $1^{\text {st }}$ and $2^{\text {nd }}$ natural frequences

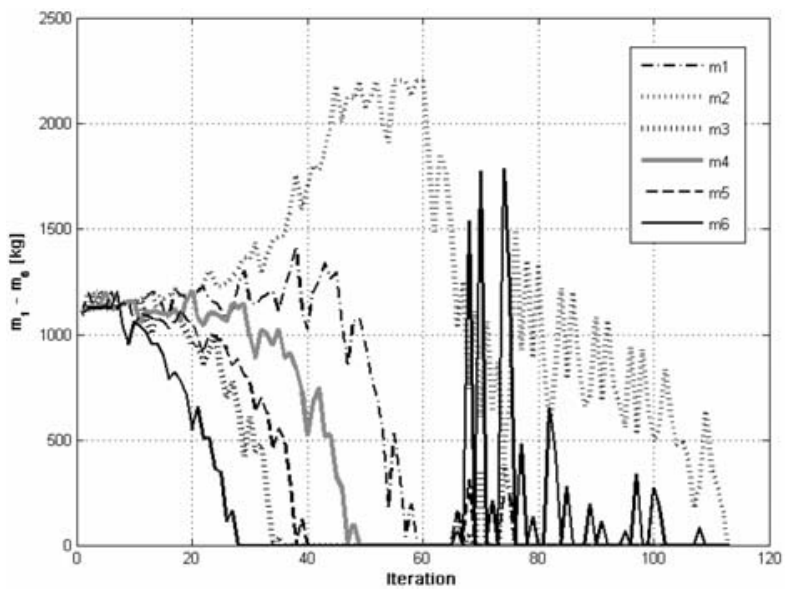

Fig. 11 History of the iteration process for cylinder masses - OPT

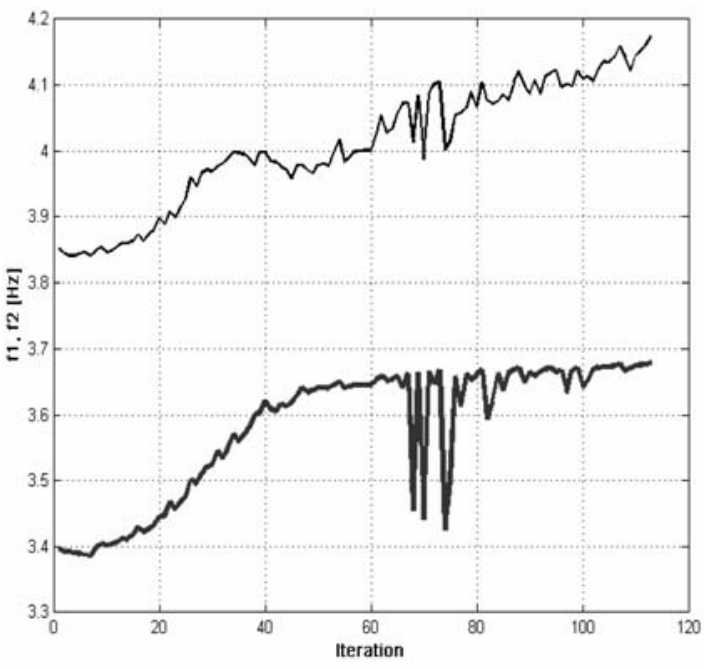

Fig. 13 History of the iteration process for natural frequences $f_{1}, f_{2}-O P T$ 
On the basis of the results obtained from the interval estimations of the FEM models spectral properties it is possible to conclude:

- the appropriateness of OPT algorithm application, which mainly due to the simplicity of the criteria function for the infimum or supremum analysis gives excellent results, in some cases even better than the MC method,

- the previous fact relates to the application of genetic searching algorithms, whose biggest advantage is their universality and particularly searching for global extremes,

- the inappropriateness of the COM1 method is demonstrated again because it shows a considerable deflection against the other methods,

- the COM2 method has a limited use but it can be suitable in combination with the OPT method because of "locating" of the solution map corner solutions.

\section{Conclusion}

The paper presents methods and their applications in an interval structural analysis. The interval arithmetic provides a new pos- sibility of the appraisal of quality and reliability of analyzed objects. Due to this numerical approach we can more authentically analyze mechanical, technological, service and economic properties of investigated structures.

The interval finite element method is a new tool for engineering problems with uncertain - inexact parameters. In the paper we investigated possibilities of the stress-strain and modal-spectral solution of finite element models with interval loading, interval geometry (interval cross section areas of the truss structure) and also interval material properties. We analyzed the interval stress response and interval natural frequency of the testing FE model.

It should be noted that the presented approaches are appropriate for linear models, the non-linearity can raise problems with solution stability and its convergence. The centre of our interest was mainly a comparison of the suggested numerical algorithms and their effectiveness evaluation.

\section{Acknowledgements}

This work has been supported by AV grant No. 4/2044/08 and VEGA grant No. 1/4099/07.

\section{References}

[1] DEKYS, V., SAPIETOVA, A., KOCUR, R.: On the reliability estimation of the conveyer mechanism using the Monte Carlo method, Proc. of COSIM2006, Krynica - Zdroj, 2006, pp. 67-74.

[2] ELISHAKOFF, I., DUAN, D.: Application of Mathematical Theory of Interval Analysis to Uncertain Vibrations, Proc. of NOISECON'94, Ft. Lauderdale, Florida, pp. 519-524, 1994.

[3] FORSSEN, P.: Interval methods I., electronic text: http://www.tdb.uu.se/kurs/optim-mn1/ht01/lectures/lec14_2.pdf

[4] KULPA, Z., POWNUK, A., SKALNA, I.: Analysis of linear mechanical structures with uncertainties by means of interval methods, Computer Assisted Mechanics and Engineering Sciences, 1998, Vol. 5, Poland, pp. 443-477.

[5] MOORE R., E.: Interval Analysis. Prentice Hall, Englewood Cliffs, New Jersey, 1966.

[6] MUHANNA, R., L., MULLEN, R. L.: Sharp enclosure for material uncertainty in solid and structural mechanics - interval based approach, 8th ASCE Spec. Conference on Probabilistic Mechanics and Structural Reliability, 2000, electronic text: http://ecivwww.cwru.edu/civil/rlm/P331.pdf

[7] NEUMAIER, A: Interval Methods for Systems of Equations. Cambridge University Press, Cambridge, 1990.

[8] RUMP, S., M.: Intlab, electronic text: http://www.ti3.tu-harburg.de/ ${ }^{\sim}$ rump/intlab/

[9] SEG_A, S., CIUPITU, L., REICH, S.: Optimization of a spring balancing mechanism for parallelogram robot mechanisms. In: 1st International Conference "Optimization of robots and Manipulators OPTIROB 2006". Predeal, Romania, 2006, pp. 69-74.

[10] ZHANG, H.: Nondeterministic Linear Static Finite Element Analysis: An Interval Approach, School of Civil and Environmental Engineering, Georgia Institute of Technology, 2005. 